\title{
Is Single Ilioinguinal approach the solution for treating all types of Acetabular Fractures- A prospective study of 54 patients.
}

\author{
Dr. Muqtadeer Ansari;M.S,DNB ${ }^{1}$, Dr. Atul K Patil ;MS, DNB ${ }^{1}$, Dr. C J Thakker \\ ;MS, $\mathrm{DNB}^{2}$, .Dr. Aditya C Pathak ; MS, $\mathrm{DNB}^{3}$, \\ ${ }^{1}$ Assistant Professor, Department of Orthopaedics, Lokmanya tilak Medical College and Hospital, Sion, \\ Mumbai , India 400022. \\ ${ }^{2}$ Ex-Professor and Head of Unit, Department of Orthopaedics, Lokmanya tilak Medical College and Hospital, \\ Sion, Mumbai , India 400022 \\ ${ }^{3}$ Assitant Professor, Department of Orthopaedics, Dr R N Cooper Hospital, Vile parle , Mumbai , India 400056
}

\begin{abstract}
We treated 54 patients with complex acetabular fractures by single ilioinguinal approach, open reduction and internal fixation using plate osteosynthesis for anterior column and lag screw for posterior column through anterior approach and prospectively followed them up for a mean period of 10.50 years (minimum 3 and maximum 27 years). We assessed patients during their serial follow-up using Harris hip score, Merle De Aubigne and Postel scrore. We correlated our results with Matta's radiological fracture reduction criteria. All patients achieved union. Reduction achieved was poor in 13\%, imperfect in $33.7 \%$ and anatomical in 53.3\% patients. Our mean Harris hip score and Merle de Aubigne score at mean follow up improved from $19.22 \& 2.57$ to $85.78 \& 15.6$ at end of mean follow up of 10.5 years. There were 2 cases of infection and 2 cases required revision and conversion to the conventional anterior + posterior approach. There were two cases of secondary osteoarthritis of hip in patients with poor reduction.

With our study we conclude that ilioinguinal approach with anterior column plating and lag screws for posterior column anteriorly offers good functional, clinical and radiological outcome for complex acetabular fractures .It must be used in experienced hands and patients selection is key to success. Comminuted posterior column fracture or fractures with more comminuted posterior column as compared to anterior column must be dealt with posteriorly. Aim must be to achieve anatomical reduction radiologically for better functional results.
\end{abstract}

Key Words: Acetabular Fractures, Ilioinguinal approach, Bicolumnar, T-shaped fracture.

\section{Introduction}

Acetabular fractures were rare but now on a rise due to high incidences of accidents, trauma and increasing awareness in treatment of acetabular fractures in last 4-5 decades. Acetabular fractures ever since have been on an upward learning curve.Precision of reduction and careful soft tissue dissection is must as in acetabular fractures it determines overall functional outcome and long term prognosis. So proper evaluation and surgical planning is must for acetabular fractures ${ }^{1}$.

There has always been debate about implication of approaches in complex acetabular fractures. Selection of proper surgical approach depending upon the acetabular fracture pattern is one of the most important factor so as to achieve proper reduction. The surgical approach for exposure of an acetabular fracture is determined by Letournel's fracture classification. The ilioinguinal approach was developed in 1965 as an anterior approach to the pelvis and acetabulum. Before this date, the Smith-Petersen incision or a modification of it called the iliofemoral approach provided the only access to the upper part of the anterior column of the acetabulum. The ilioinguinal approach provides total and complete access to the anterior column from the sacroiliac joint to the pubic symphysis. Both column fractures typically can be treated through the ilioinguinal approach. Damage to neurovascular bundle, lymphatics, Heterotopic ossification etc. are known complication. In our study we used ilioinguinal approach for all patterns of acetabular fractures barring few and fixed posterior column irrespective of comminution with long Cancellous screws under C-Arm guidance. We have shown good results and less complication rates using this approach in our case series.

\section{Material \& Methods}

This study is a prospective, open labeled observational clinical study, approved by the institutional ethics committee and consented by patients participating. We selected all patients operated for acetabular fractures by ilioinguinal approach with anterior plating for anterior column and lag screws for posterior column anteriorly. 54 patients were selected. We had 33 patients with bicolumnar fractures, 14 patients with T-shaped fractures, 3 patient with transverse fracture and 4 patient with anterior column fracture with posterior hemitransverse fracture. 
Inclusion criteria-

- All types of fracture pattern as per Letournel et al, except isolated posterior column or posterior wall fractures.

- Only isolated acetabular fractures were selected.

- Only acute trauma cases were selected .

- Those patients operated within first two weeks of injury were selected.

\section{Exclusion Criteria-}

- Posterior wall fracture with any fracture pattern

- Isolated anterior wall and anterior column fracture or both.

- Isolated posterior column fracture.

- Any fracture with intra-articular fragment or cases of fracture dislocation.

- Any patient of polytrauma with associated other fractures or any other significant head, chest or Abdominal trauma.

Hypotensive Spinal- Epidural Anaesthesia was given and the blood pressure was maintained at 90 / 60 $\mathrm{mm} \mathrm{Hg}$ throughout the surgery. Femoral block was given pre-operatively to facilitate positioning of patient for anaesthesia, and also a measure of post-operative pain relief for the patient.

Surgery was performed in supine position which could be converted to an floppy lateral position when needed. The ilioinguinal approach was developed by Emile Letournel based on cadaveric dissections to provide anterior access for fractures of the acetabulum. A curved incision beginning posterior to the gluteus medius pillar and extend past the midline $2 \mathrm{~cm}$ proximal to the symphysis.In case of thin individuals, placing the lateral limb of the incision distal to the ilioiliac crest may avoid a tender scar.Begin by exposing the internal iliac fossa.

Release the external oblique insertion onto the iliac crest, taking care to leave a thick fascial/ periosteal cuff to facilitate repair.Initially, leave the tissues attached to the anterior superior iliac spine.In continuity with this release, expose the internal iliac fossa subperiosteally by mobilizing the iliacus muscle.Pack the fossa with a sponge.Next, the external oblique aponeurosis is incised from the anterior superior iliac spine (ASIS) to the lateral border of the rectus sheath, passing cranial to the external inguinal ring. The spermatic cord (or round ligament) is mobilized in the medial aspect of the wound.Medially the transversus abdominis is then released from the inguinal ligament, usually taking 1-2 $\mathrm{mm}$ of the ligament with the tendon. This release begins at the anterior superior iliac spine and progresses medially to the conjoint tendon of the internal oblique, and the pubic tubercle.Care must be taken during this portion of the procedure to protect the ilioinguinal nerve which normally lies just proximal to the inguinal ligament after penetrating the abdominal wall.The lateral cutaneous nerve of the thigh is usually encountered just deep to the conjoint tendon (of the internal oblique and the transversus abdominis) approximately $1-2 \mathrm{~cm}$ medial to the anterior superior iliac spine.This nerve can usually be preserved if it is mobilized as it exits the abdominal wall and enters the fascia of the thigh.The anterior aspect of the iliopsoas muscle is thus exposed in the lateral portion of the wound with the femoral nerve lying on its anteromedial surface. The iliopectineal fascia is delineated by careful retraction of the femoral vessels medially and the femoral nerve and iliopsoas laterally.It is then divided distally, under direct visualization, down to the pubic root.The iliopsoas is then retracted laterally, exposing the fascial attachment to the pelvic brim which can be divided safely thus entering the true pelvis can be entered from the internal iliac fossa.Dissection around the iliac vessels should be minimized. This limits risk of vascular injury and also preserves the path of the primary lymphatic trunk to the lower extremity which passes medial to the vein.

The 3 windows of the ilioinguinal approach can now be fully exploited. The first window medial to external iliac artery \& vein encompasses the entire internal iliac fossa from the sacroiliac joint posteriorly to the iliopectineal eminence anteriorly.This window is optimized with hip flexion to relax the iliopsoas.Medial retraction usually requires placement of retractors on the quadrilateral surface. The second window between external iliac vessels and the iliopsosas provides access to the pelvic brim and quadrilateral surface from the sacroiliac joint to the lateral third of the superior pubic ramus. Medial retraction of the femoral vessels should be gentle and must be carefully monitored.The third window medial to iliopsoas (iliopectineal fascia) can be developed in a number of different ways. The most limited of these leaves the ipsilateral rectus insertion attached and visualization is provided between the rectus and the spermatic cord (or round ligament).Alternatively, if the fracture pattern requires, the entire medial portion of the superior ramus and symphysis can be visualized by release of the ipsilateral rectus insertion.

The same visualization can be achieved by leaving the rectus attached and splitting the rectus heads in the midline. With the rectus still attached, retraction is carried out posterior to the rectus with a Hohmann retractor placed along the superior ramus. All three variations require that the bladder be protected. This can be achieved by packing the prevesicular space with a sponge after the bladder has been identified by palpation of the urinary catheter bulb.It is frequently useful for the operating surgeon to perform the third window exposure from the opposite side of the table for optimum visualization.This provides a view from the symphysis looking 
laterally along the superior ramus and pelvic brim. This exposure can be developed further to include the entire pelvic brim and quadrilateral surface.The third window developed in this manner provides surgical access equivalent to the modified Stoppa approach. Before closure, one may place drains in the space of Retzius and anterior internal iliac fossa.

\section{Method of posterior column reduction}

Reduction of the posterior column requires lateral and anterior traction of the hip either through Schanz pin in the femoral head or a specially designed clamp with one end on the outer surface of the iliac wing and another through the lateral window of the ilioinguinal exposure over the quadrilateral plate or the posterior column, accurately holding the reduction of the posterior column to the anterior.A small supplemental bone hook gently slide down the quadrilateral plate can hook the ischial spine and pull up the posterior column up to the anterior column . On reduction $3.5 \mathrm{~mm}$ lag screw is inserted through the pelvic brim superior to the acetabulum into the posterior column. Care must be taken to avoid intra articular placement of lag screws keeping in the mind the relative location of the acetabulum under the iliopubic eminence and inferior to the anterio inferior iliac spine. To best prevent joint penetration this screws should parallel the quadrilateral surface aiming towards the ischial spine.from a more proximal starting point in the iliac fossa, screws aimed at the ischial tuberosity are used obtaining fixation of posterior column with long screws.
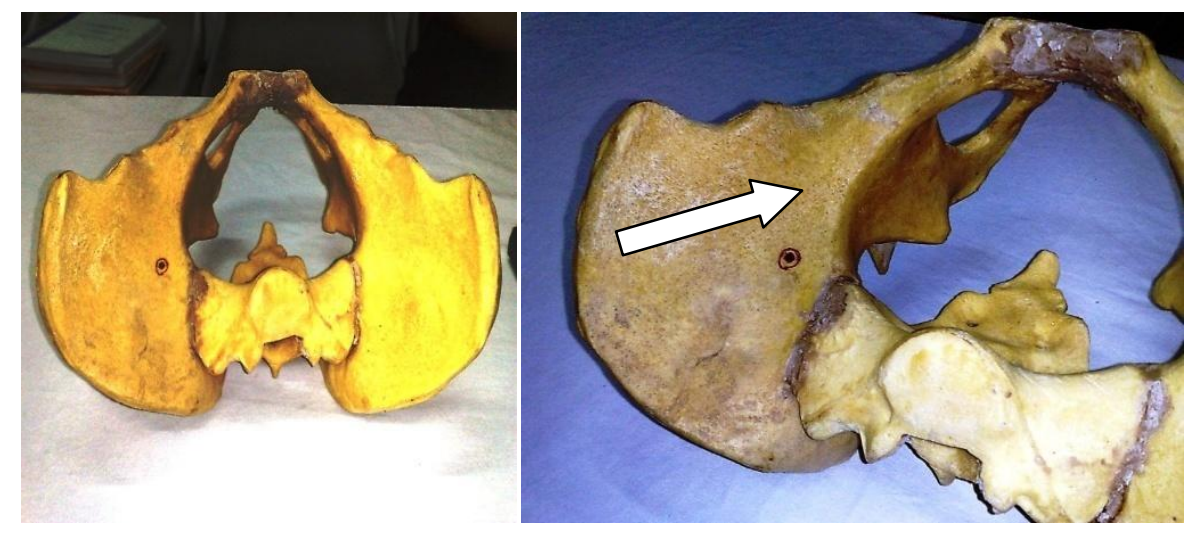

Layered closure then begins with repair of the conjoint tendon to the inguinal ligament. A careful fascial repair restores the floor of the inguinal canal.The external oblique aponeurosis and the rectus sheath are then repaired, followed by secure reattachment of the abdominal wall origin to the iliac crest, in the lateral portion of the incision. A hernia-free repair, and avoidance of entrapment of the spermatic cord should be achieved.Subcutaneous drains may be inserted.Finally, perform an appropriate subcutaneous and skin closure.

Post-operative protocol: Post-operatively, drain was kept for $<48$ hours after the surgery. Sterile dressing was done on post-op day 2. Intravenous antibiotics were continued at 12 hourly intervals for the duration of 5 days post-operatively and then no oral antibiotics were given. Suture removal was usually done on 14th postoperative day. Mobilisation and physiotherapy protocols were given to the patients on individualized basis as per the extent of surgery and soft tissue dissection.

Patients had undergone clinical and radiological evaluation at 6 weeks, 3 months, 6 months, 1 year and then annually after the surgery. We have evaluated final outcomes in terms of final displacement as measured in post-operative radiographs. $0-1 \mathrm{~mm}$ displacement was considered to be anatomic, $2-3 \mathrm{~mm}$ displacement as satisfactory result and $>3 \mathrm{~mm}$ displacement was considered as unsatisfactory.

At each follow up, range of motion at hip joint, Harris hip score, and Visual analogue scores were noted. The outcomes of Harris hip score were classified as excellent (91-100\%), good (81-90\%), fair (71-80\%) and poor $(\leq 70 \%)$.In our series, VAS score was classified as excellent (0-2), good (3-6) and poor (7 -10).

\section{Statistical Analysis}

Statistical analysis was done on Microsoft excel sheet, Minitab using SPSS 25 software, Null hypothesis and Paired t test/ One way ANOVA test. It was done using Microsoft excel 2007 and Microsoft word 2007.

\section{Results}

Results of our study were encouraging and were in favor of using Single ilioinguinal approach using lag screws for posterior column fixation and anterior column plating.

Demography and Fracture pattern- 
Our study include total 54 patients and were followed up for a mean period of 10.5 years (minimum 3 years and maximum 27 years). Of them 9 patients were female and 45 were male. Patients of all age group were included, most of them were young with mean age of 37.6 years.( minimum 18 years and maximum 72 years).

30 patients had sustained right sided acetabular fractures and 22 had sustained Left sided fractures. 2 Patients had sustained bilateral fractures. Only fresh fractures were included with duration between trauma and surgery (mean) being 83.70 hours (minimum 24 hours and maximum 10 days). 43 patients had no comorbidities, rest 11 patients had diabetes and hypertension but its effect on final outcome in our study was not significant.

\section{Fracture pattern- Table I.}

\section{Radiological Assessment -}

Assessment of Fracture fixation was done using JM Matta's criteria. We had achieved anatomical reduction in 29 patients, Imperfect (acceptable) reduction in 18 patients and poor reduction in 7 patients. $100 \%$ anatomical reduction was achieved in anterior + posterior hemitransverse and Transverse fracture type. It was difficult to get anatomical reduction in Bicolumnar and $\mathrm{T}$ shaped fracture pattern - 36.4\% and $71.4 \%$ respectively. Poor reduction was present in $21.2 \%$ of bicolumnar fracture indication difficulty in achieving reduction. Both Harris hip score and Merle De Aubigne scores were highest when anatomical reduction was achieved .The difference in both Harris hip and Merle De Aubigne scores were significant between anatomical reduction achieved and poor reduction (table II) indicating good prognosis and functional results when anatomical reduction was achieved. Two out of 7 patients with poor reduction by single approach required revision surgery and refixation of comminuted posterior column by posterior approach. Two patients with imperfect reduction had infection.

Hip Scores-

The overall Mean Scores both Harris Hip Score and Merle De Aubigne at follow up of 10.5 years were 85.78 and 15.50 respectively from pre-operative mean of 19.22 and 2.57 . This indicated good functional results at end of follow up. The difference of score distribution with respect to fracture pattern in our study was not found to be significant. The distribution of scores with respect to fracture pattern had been summarized in Table III. Other variables-

The estimated mean blood loss by our method was $511 \mathrm{ml}$ ( minimum being $300 \mathrm{ml}$ and maximum $1000 \mathrm{ml}$ ). Estimated mean surgical time was 2.69 hours( minimum being 2 hours and maximum being 4.5 hours). Both Blood loss and surgical time had no significant association with the final functional outcome or the reduction achieved. But two cases which had got infected were operated for about (4 hours) longer duration.

\section{Complications}

We had encountered with certain complication, none causing fatalities in our study. There was one case of intra-operative breakage of $\mathrm{K}$ wire within the substance of bone, which needed removal. Bicolumnar fractures with comminuted posterior column were the most difficult to reduce but statistical association could not be attributed. Seven of the bicolumnar fractures had poor anatomical reduction. Two of them with comminuted posterior column required revision in form of open reduction and internal fixation with plating by posterior approach. Of the remaining of the 5 patients with poor reduction 3 followed up with us for about 5 years, showed changes of secondary arthritis of hip but none of the cases in our series had avascular necrosis of femoral head during their follow up. Two patients with imperfect reduction had got infected, one of which detected infected on post-operative day ten presented with severe wound tenderness and sero-purulent discharge healed with debridement, wound wash and antibiotic regime as per culture sensitivity report. Other case presented to us with infection 13 months post -operative for whom we did implant removal as fracture was healed and gave wound wash with debridement.

\section{Discussion}

The choice of surgical approach in the management of complex fractures of the acetabulum will probably continue to evolve as instruments and imaging techniques improve, as well as with changes in surgical training. While the selection of the appropriate approach is clear for most of the simple patterns of fracture in the Letournel classification, the choice of exposure in the complex patterns is a matter of the surgeon's choice, based on his individual experience and training. This makes comparison of outcome based on the pattern of fracture difficult. The functional outcome after surgery for fractures of the acetabulum correlates with the accuracy of the reduction which is dependent, to a significant degree, on the selection of the best surgical exposure based on the pattern of the fracture. As instrumentation and techniques have improved, more such fractures have been managed through either the ilioinguinal or Kocher-Langenbeck approach. The extended iliofemoral approach is the most challenging of the standard three approaches and we recommend use of the ilioinguinal or Kocher-Langenbeck exposures whenever possible. We have used in our study a single 
ilioinguinal approach, plating for anterior column and screws should be parallel to the quadrilateral surface aiming towards the Ischial spine.From a more proximal starting point in the iliac fossa, screws aimed at the ischial tuberosity are used obtaining fixation of posterior column with long screws. One screw is inserted from the posterior part of the iliac fossa through the lateral window and the other through the medial window from the iliopectineal eminence close to the pelvic brim. This type of fixation has not been associated with secondary displacement and appears sufficient to resist the stress of immediate active movement ${ }^{4}$.

The timing of operation, although a key factor in optimizing the outcome of acetabular surgery and for management to be effective, definitive surgery should be performed as soon as possible. Bosse et al (1988)used an extended or modified extended iliofemoral approach, and reported a $50 \%$ incidence of Brooker III and IV heterotopic ossification, with $30 \%$ of these groups having ankylosis of the hip. Matta (1992), using an extended iliofemoral approach, reported a $12 \%$ incidence of disabling heterotopic ossification. Because of other variables such as head injury, soft-tissue trauma and timing of surgery, it is impossible to draw firm conclusions about the association between the approach and subsequent heterotopic ossification, but our results suggest that a nonextensile approach decreases the risk of disabling changes.' In our study we had selected all acute cases with isolated acetabular fractures. Also in our study we had no incidences of heterotopic ossification may be because all cases were acute and operated early with a single ilioinginal approach.

Meralgia Paraesthetica is nearly always seen during an ilio-inguinal approach. These three patients found their disability irritating rather than a great burden. In an ilio-inguinal approach, the lateral cutaneous nerve of the thigh is under threat, especially during difficult reductions. Significant weakness of adduction of the hip in the use of the other approaches has been noted in $26 \%$ of patients, which may be due either to the injury or the iatrogenic stretching of the obturator nerve during exposure of the quadrilateral plate. Traction injuries of the lateral femoral cutaneous nerve have been reported in $5 \%$ of patients ${ }^{12}$ and of the femoral nerve in $4 \%$ of patients.Finally, postoperative hernia formation has been described in $2 \%$ (one in 55) to $3.5 \%$ of cases (two of 57). Sciatic nerve injury was more common in ilioinguinal approaches in both groups, likely due to reduction techniques for the posterior column performed with the hip flexed, placing the sciatic nerve under tension.' In our series we did not encounter with any issues of neurological or vascular insult, neither did we come across any case of hernia during our follow-up.

The ilioinguinal approach was described for the management of acetabular fractures involving predominantly the anterior column but in our hands does not allow a good view of the impacted acetabular dome fragments, resulting in a suboptimal reduction of the fracture. We have found that the approach described here allows for a more medial approach so that reduction forces can be better applied along the pelvic brim and orthogonal to the fracture displacement. The choice of the ilioinguinal approach has great advantage in the treatment of anterior column fractures with associated posterior hemitransverse fractures. In our study too we found that single ilio-inguinal approach was satisfactory for $\mathrm{T}$ shaped, Anterior column+posterior hemitransverse fractures and for transverse fracture in order to achieve anatomic reduction. Bicolumnar fractures especially with multifragmented comminuted posterior column were the most difficult to deal with, and results in those cases were not satisfactory.

Certain studies have shown that the stiffness of the lag screw method was $39 \%$ higher than that of the plating method. In addition, the plate and screw method provided significantly greater maximum strength than the screw and wire technique. The quadrilateral plate seemed to be the weakest area of fixation because $83 \%$ of the implant failures occurred in this region. In patients in whom the risks of formal open reduction and internal fixation of acetabular fractures outweigh the possible benefits, lag screw fixation provided relatively greater stiffness, which may account for its clinical success. In acetabular fractures with sufficiently large fragments, screw fixation with $3.5 \mathrm{~mm}$ cortical screws proved satisfactory. In very comminuted fractures or where there is poor patient compliance an additional buttress plate should be used.Percutaneous lag screw fixation of appropriate transverse acetabular, non comminuted bicolumnar fractures, non comminuted $\mathrm{T}$ shaped fractures and posterior hemitransverse fractures is a viable option.' From our study too we inferred the same that lag screw fixation in viable option for achieving good functional results provided comminution of posterior column is less and large fracture fragments are present.

There are studies which have shown use of minimal ilioinguinal approach as an acceptable approach for both column fixation. By this approach there is an indirect access through the second window to quadrilateral surface and posterior column to the level of ischial spine. Reduction technique and implants i.e lag screw for posterior column and plate for quadrilateral surface are the standard ones. Although we have not used minimally invasive approach in our study but based on our success rate of reduction we agree that ilioinguinal approach in experienced and skilled hands is a versatile approach for almost all fractures of acetabulum. One of the major disadvantages with this approach is no access to internal aspect of hip joint directly or indirectly so we excluded all such fractures pre-operatively. We also find that as patient is in floppy lateral position, use of image intensifier becomes easy, and also there is ease of applying traction and manoeuvring the ipsilateral lower limb helped significantly to achieve reduction of fracture. In our study we found that it was most difficult to reduce $\mathrm{T}$ 
shaped fractures and bicolumnar fractures with greater posterior column communition. In our study only $36.6 \%$ of bicolumnar fractures and $71.4 \%$ of $\mathrm{T}$ shaped fractures were reduced anatomically. Results of our study were comparable to study by Matta. ${ }^{7}$ Matta also had suggested that it is relatively easy to fix bicolumnar fractures as compared to T- shaped fractures as in former joint capsule and acetabular labrum are firmly attached to both anterior and posterior columns as they act as hinge while reducing the fracture.. But we ,from our experience would further say that this statement holds true when columns are less comminuted especially posterior column.

In our study we achieved anatomical reduction in $53.3 \%$ of patients and acceptable/imperfect reduction in $33 \%$ of patients. Over all $86 \%$ of patients in our studies had good outcome which is comparable to studies by Chiu et al and Deo et al.'. In our case series, we had no incidences of avascular necrosis of femoral head as we had not included cases of acetabular fractures with hip dislocation. Also it can be attributed to use of ilioinguinal approach as it does not violate the blood supply of femoral head which comes from posteriorly. Overall incidences of avascular necrosis of femoral head in acetabular fractures are between 3-10\%.' . In our studies we encountered very less infection about $0.3 \%$ which is comparable to other studies ${ }^{20}$, which show overall $0-3 \%$ of infection rates by single approach. On the Contrary there is $5-12 \%$ infection rates associated with extensile or dual approaches. ${ }^{21}$,

Overall to summarize there are not many studies in literature to support both column fixation by single conventional ilio-inguinal approach. Very few studies have actually proven it. ${ }^{4,7 .}$. Furthermore there have been many modifications of ilio-inguinal approach described till date. We would stick to the original approach described by Letournel et al, as there have been few studies evaluating the outcome and reproduction of favourable results and versatility of those approaches in various types of acetabular fractures. ${ }^{17}$,

We thoroughly agree to Letournal et al that the ilioinguinal approach is justified for the following reasons: (1) it is an anatomic approach that can restore the anatomy of the inguinal canal; (2) it does not disturb the abductor mechanism, and the recovery of the hip mobility is faster and postoperative pain significantly reduced; (3) it eliminates the associated postoperative risks of hip mobilisation associated with trochanter osteotomy or tenotomy of the gluteal tendons. The ilioinguinal approach does not involve a capsulotomy, which also may be an advantage in regaining hip motion. The ilioinguinal incision is cosmetic and heals quickly with no associated injury to major muscle groups. It significantly reduces the risk of ectopic bone formation. The results achieved through the ilioinguinal approach may be superior to reduction of these associated fracture patterns using double incisions.

None

\section{Acknowledgements}

Conflict of interest - None

Legends for Tables -

Table I - Fracture Pattern

Table II - Mean values of Harris hip scores and Mearle De Aubuigne and their difference.

Table III- Score distribution with respect to fracture pattern.

Legends For Figures -

Figure I - A, B, C- Showing Antero-posterior And Judets X-ray views, D, E,F- Showing CT scan Axial and 3-D Reconstruction Images of Bicolumnar Fracture pattern, G,H, I - Showing Immediate Post Operative Antero-posterior and Judets View of Open Reduction Internal Fixation Done For Bicolumnar fracture by ilio-inguinal approach.

Figure II - A,B,C, D- 5 year follow up x-ray Antero-posterior and Judets view of The same bicolumnar fracture with single ilio-inguinal approach. E,F- Clinical Images of patient sitting cross-legged and Squatting comfortably.

Figure III - A,B,C - Showing antero-posterior view and Judets view of acetabulat fracture. D,E,FShowing CT images Axial cut, sagittal cut and 3-D Reconatruction of fracture pattern conforming $\mathrm{T}$ shaped fracture. G,H,I - Showing Immediate post-operative Antero-posterior view with good reduction by single ilioinguinal approach.

\section{References}

[1]. Rommens PM:(1999) Acetabulum fractures. Unfallchirurg, 102:589-590

[2]. Hirvensalo E, Lindahl J, Kiljunen V: (2007), Modified and new approaches for pelvic and acetabular surgery. Injury, 38:431-441

[3]. Matta JM.-Fractures of the acetabulum:(1996) accuracy of reduction and clinical results inpatients managed operatively within three weeks after the injury. J Bone Joint Surg[Am];78-A:1632-45.

[4]. Letournel E - (1993)The treatment of acetabular fractures by ilioinguinal approach. Clin Orthop Relat Res , July;(292):62-76

[5]. Weeks after injury.J bone J Surg. NOVEMBER, VOL. 78-A, NO. 11, Helfet DL, Schmeling GJ.(1994)

[6]. Management of complex acetabular fractures through single non extensile exposures.Clin Orthop ;305:58-68. Matta JM.- (1994)

[7]. Operative treatment of acetabular fractures through the ilioinguinal approach: a 10-year perspective.Clin Orthop ;305:10-19. 
[8]. Madhu R, Kotnis R, Al-Mousawi A, et al.(2006) Outcome of surgery for reconstruction of fractures of the acetabulum: the time dependent effect of delay. J Bone Joint Surg [Br] ;88-A:1197-203.

[9]. Bosse MJ, Poka A, Reinert CM, et*1.(1988) Heterotopic ossification as a complication of acetabular fracture : prophylaxis with low-dose irradiation. J Bone Joint Surg [Am] ; 70-A :1231-7.

[10]. Matta JM. Symposium : (1992)Management of acetabular fractures Part II. Contemporary Orthopaedics, 25:389-412.

[11]. Briffa, Pears, Hill et al -(2011) Outcome of acetabular fracture with 10 years follow up. J Bone Joint Surg [Br] ;93-B:229-36.

[12]. Sagi HC, Afsari A, Dziadosz D. (2010) The anterior intra-pelvic (modified rives-stoppa) approach for fixation of acetabular fractures. J Orthop Trauma 2010;24:263-270

[13]. John G, Julia S, Roy W et al - (2002) Iatrogenic Nerve Injury in Acetabular Fracture Surgery: A Comparison of Monitored and Unmonitored Procedures. Journal of Orthopaedic Trauma: May - Volume 16 - Issue 5 - pp 297-301

[14]. Keel, Ecker,Bastian et al -(2012) Pararectus approach for anterior intrapelvic management of acetabular fractures. Bone Joint Surg Br;94-B:405-11

[15]. Sanjitpal S, Robert Z, William K et al -(2001) Comparative strenght of three method of fixation for transverse acetabular fractures. Clin Orth Related Research November - Volume 392 - Issue - pp 433-441

[16]. Stockle U, Haas N, Hoffman R- (2000)Screw Fixation for acetabular fractures. International Orthopaedics July, Volume 24, Issue 3, pp 143-147

[17]. Wolf $\mathrm{H}$, Weiland $\mathrm{T}$, Pajenga $\mathrm{G}$ et al-(2007) Minimally invasive ilioinguinal approach to acetabulum.Injury,Int $\mathrm{J}$ Care Injured;38;1170-76

[18]. Chiu FY, Chen CM, Lo WH- (2000) Surgical treatment of displaced acetabular fractures- 72 cases followed for 10 (6-14) years. Injury 2000;31;181-185.

[19]. Deo SD, Tavares SP, Pandey RK et al-(2001) Operative management of acetabular fractures in oxford. Injury 2001;32;581-586.

[20]. Helfet D1, Schmeling GJ- (1994)Management of Complex Acetabular fractures through single non-extensile exposures. Clin Orthop Res Relat;305;58-68.

[21]. Kaempffe FA, Bone LB, Border JR- (1991)Open Reduction and internal fixation of acetabular fractures; Heterotopic ossification and other complication of treatment. J Orthop Trauma;5;439-45.

[22]. Liebergall M, Mosheiff R, Low J et al - (1999)Acetabular fractures Clinical outcome and surgical treatment. Clin Ortho Res Relat; 366;205-16.

[23]. Alonso JE, Davila R, Bradley E-(1994) Extended iliofemoral vs triradiate approaches in management of acetabular fractures. Clin Orthop Res Relat;305;81-7.

[24]. Kumar, Shah, Clayson- (2005)Operative management of acetabular fractures by single approach-73 cases.Injury;36,605-612.

[25]. Timothy GW, Mast JW-(1994) Extended ilioinguinal approach for specific both column fracture acetabulum. Clin Orthop Res Relat; 305;106-111.

[26]. Kloen P, Siebenrock KA, Granz R-(2002) Modification of ilio-inguinal approach;16;586-93.

Figure I -
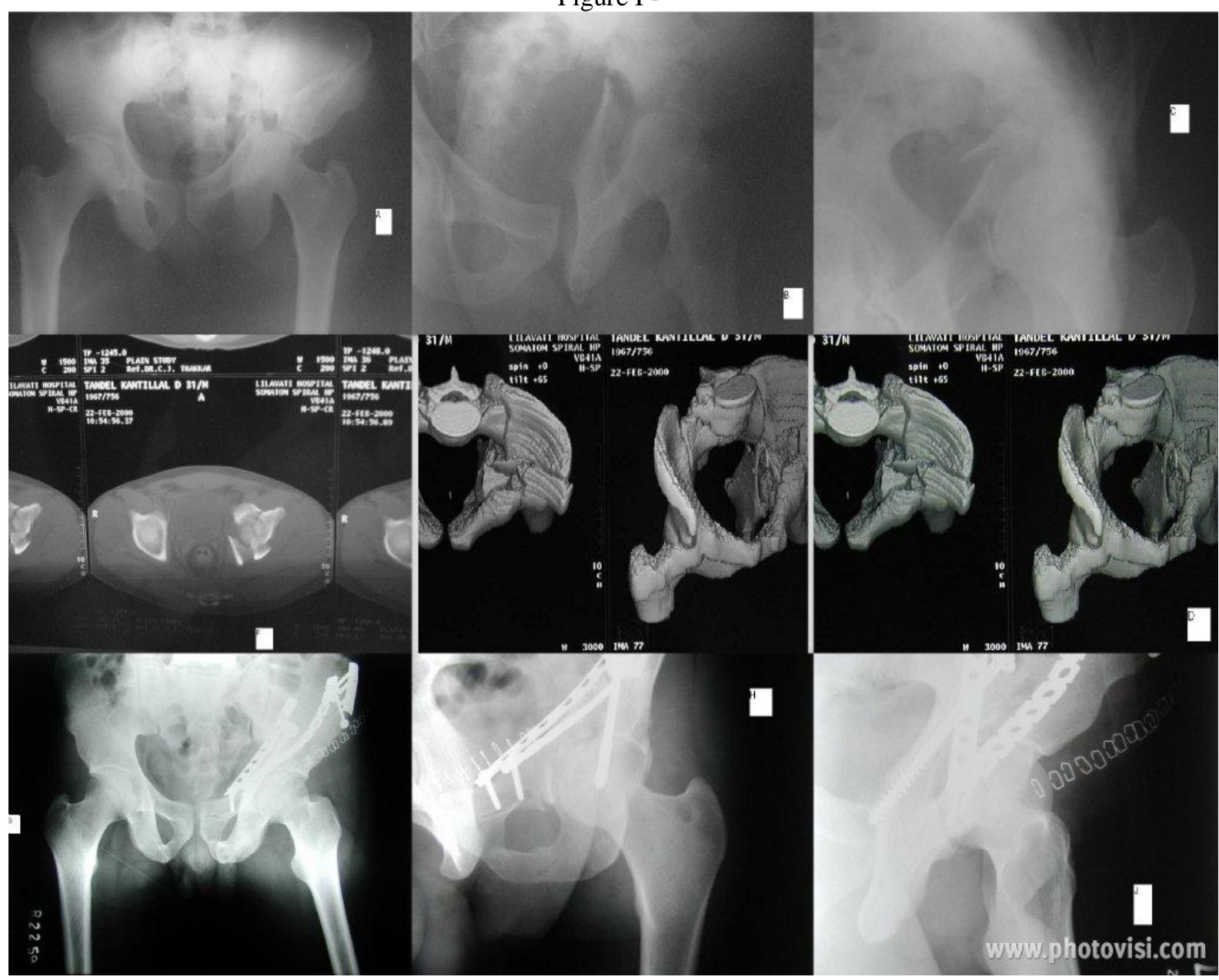
Figure II -
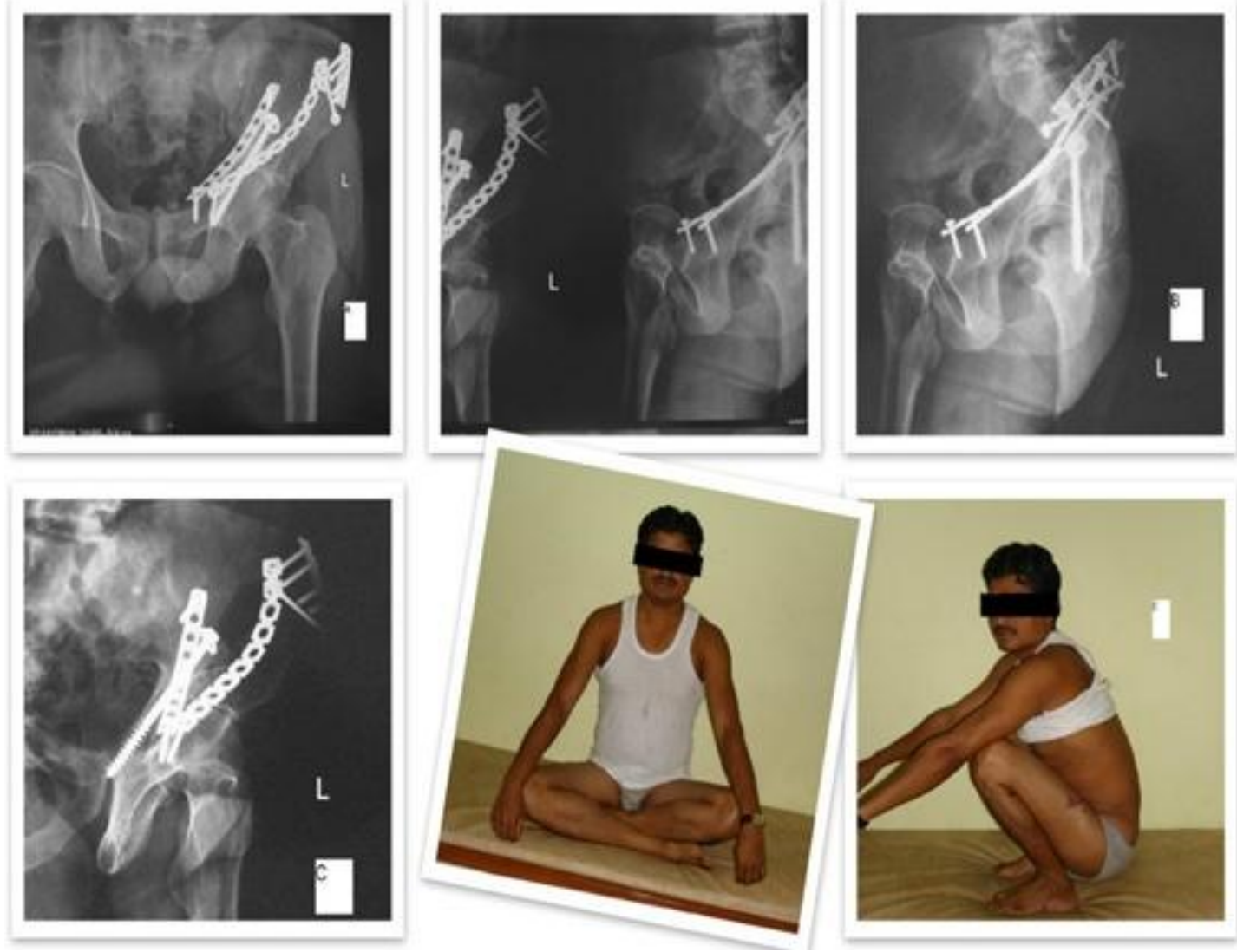

Figure III -

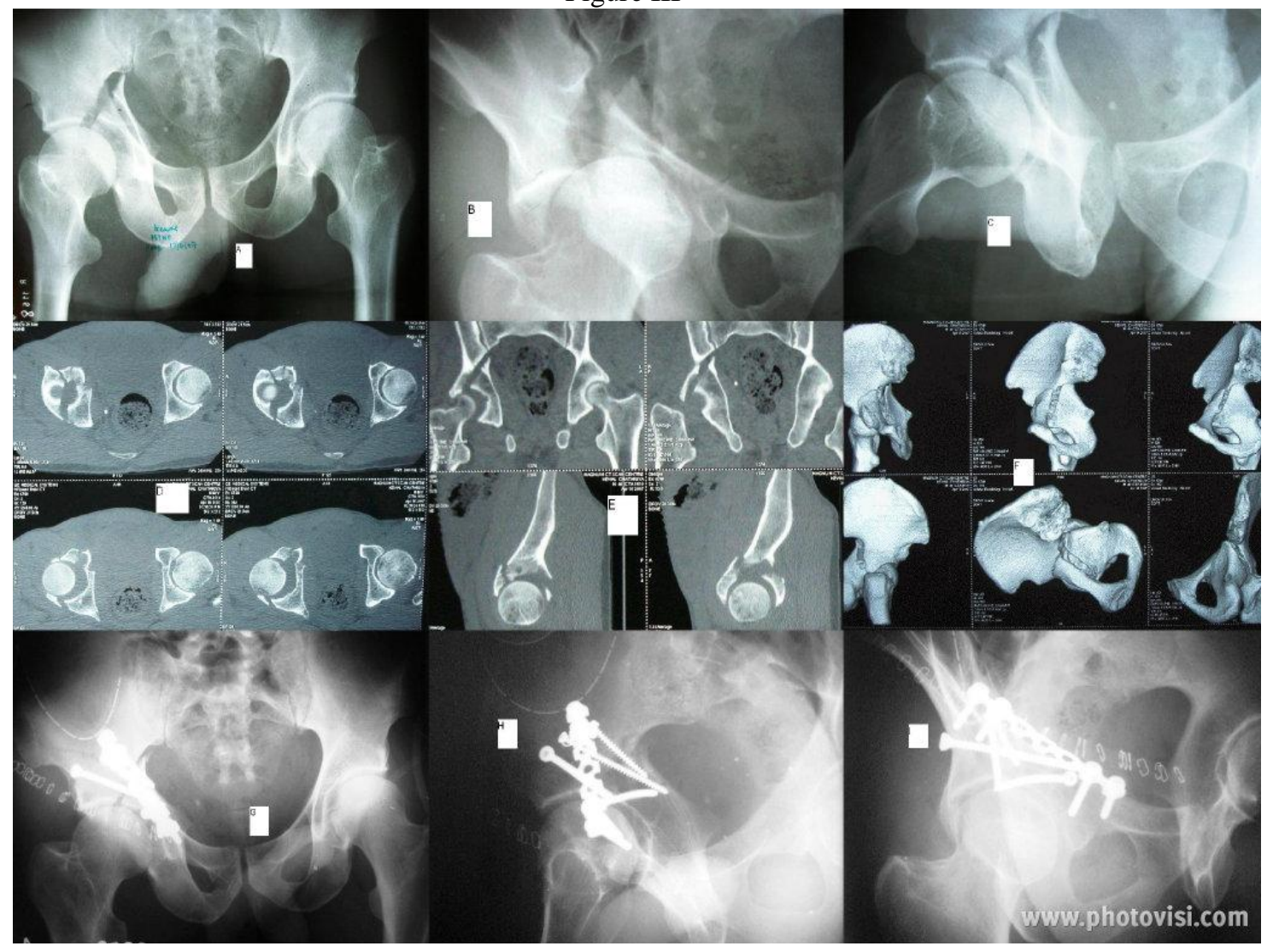


Table I

\begin{tabular}{|l|c|c|}
\hline Type of fracture & No. & Percentage \\
\hline Bicolumnar & 33 & $61.1 \%$ \\
\hline T- shaped & 14 & $25.9 \%$ \\
\hline Anterior + posterior hemitransverse & 4 & $7.4 \%$ \\
\hline Transverse & 3 & $5.6 \%$ \\
\hline Total & 54 & $100.0 \%$ \\
\hline
\end{tabular}

Table II -

\begin{tabular}{|c|c|c|c|c|c|c|c|}
\hline & & $\begin{array}{l}\text { Anatomica } \\
1\end{array}$ & Imperfect & Poor & $\begin{array}{l}\text { Differenc } \\
\mathrm{e}\end{array}$ & P-value & Result \\
\hline \multirow[t]{4}{*}{$\begin{array}{l}\text { HARRIS } \\
\text { HIP } \\
\text { SCORE }\end{array}$} & Mean & 71.10 & 65.89 & $\begin{array}{l}49.4 \\
3\end{array}$ & $\begin{array}{l}\text { Anatomic } \\
\text { vs } \\
\text { Imperfect }\end{array}$ & 0.048 & $\begin{array}{l}\text { Significan } \\
\mathrm{t}\end{array}$ \\
\hline & Minimum & 60 & 52 & 44 & $\begin{array}{l}\text { Anatomic } \\
\text { vs poor }\end{array}$ & $1.29 \mathrm{E}-08$ & $\begin{array}{l}\text { Significan } \\
\mathrm{t}\end{array}$ \\
\hline & $\begin{array}{l}\text { Maximu } \\
\mathrm{m}\end{array}$ & 82 & 84 & 58 & $\begin{array}{l}\text { Imperfect } \\
\text { vs poor }\end{array}$ & $1.17 \mathrm{E}-05$ & $\begin{array}{l}\text { Significan } \\
\mathrm{t}\end{array}$ \\
\hline & $\begin{array}{l}\text { Standard } \\
\text { Deviation }\end{array}$ & 6.52 & 8.42 & 5.97 & & & \\
\hline \multirow{4}{*}{$\begin{array}{l}\text { MEARLE } \\
\text { DE } \\
\text { AUBUIG } \\
\text { NE }\end{array}$} & Mean & 14.10 & 12.44 & 9.29 & $\begin{array}{l}\text { Anatomic } \\
\text { vs } \\
\text { Imperfect }\end{array}$ & $<=0.05$ & $\begin{array}{l}\text { Significan } \\
\mathrm{t}\end{array}$ \\
\hline & Minimum & 12 & 11 & 6 & $\begin{array}{l}\text { Anatomic } \\
\text { vs poor }\end{array}$ & $<=0.05$ & $\begin{array}{l}\text { Significan } \\
\mathrm{t}\end{array}$ \\
\hline & $\begin{array}{l}\text { Maximu } \\
\mathrm{m}\end{array}$ & 16 & 14 & 12 & $\begin{array}{l}\text { Imperfect } \\
\text { vs poor }\end{array}$ & $>0.05$ & $\begin{array}{l}\text { Not- } \\
\text { Significan } \\
t\end{array}$ \\
\hline & $\begin{array}{l}\text { Standard } \\
\text { Deviation }\end{array}$ & 1.24 & 0.86 & 2.29 & & & \\
\hline
\end{tabular}

